\section{Liquor, xanthochrom}

T. O. Kleine

Institut für Laboratoriumsmedizin und Pathobiochemie, Molekulare Diagnostik Standort Marburg Referenzlabor für Liquordiagnostik, UKGM Universitätsklinikum Gießen und Marburg, Marburg, Deutschland

Synonym(e) Gelber Liquor; Liquor-Bilirubin; Liquor-Oxyhämoglobin; Liquor-Methämoglobin

Englischer Begriff xanthochromic cerebrospinal fluid (CSF)

Definition Gelbe Farbe von CSF mit rötlicher oder bräunlicher Tönung.

Beschreibung Xantochromie in CSF wird erzeugt durch Bilirubinoide, gebunden an Proteine in Albumin- und Präalbumin-CSF-Fraktion, durch $\mathrm{Fe}^{++}$-Oxyhämoglobin und $\mathrm{Fe}^{+}$
${ }^{++}$-Methämoglobin, gebunden an Proteine in $\beta_{1}$-GlobulinCSF-Fraktion.

Bilitubin ist ein Neurotoxin, dessen Wirkung durch Proteinbindung vermindert wird: Ca. $25 \%$ der xanthochromen Liquorproben reagieren positiv mit Bilirubin-Teststreifentests ( $\triangleright$ Liquor-Bilirubin, Teststreifen-Test) bei Gefäßerkrankungungen, Verletzungen oder Tumoren des Zentralnervensystems (ZNS). Oxyhämoglobin wird bei ca. $540 \mathrm{~nm}$, Methämoglobin bei ca. $410 \mathrm{~nm}$ spektrophotometrisch nachgewiesen, was diese ZNS-Erkrankungen nicht differenzieren kann.

\section{Literatur}

Hansen TWR (2001) Bilirubin brain toxicity. J Perinatol 21:S48-S51 Kleine TO (1980) Liquordiagnostik: Untersuchungen mit Schnelldiagnostica. Untersuchungen zur Adsorption von Proteinen in Glas- und Kunststoffröhrchen. J Clin Chem Clin Biochem 18:7-11

Kjellin KG (1986) Xantochromic compounds in the CSF. In: Schmidt RM (Hrsg) Der Liquor cerebrospinalis. Untersuchungsmethoden und Diagnostik, Bd 2. G Fischer, Stuttgart/New York, S 634-651 